\title{
A CASE OF ATYPICAL COLOBOMA ASSOCIATED WITH ABNORMAL RETINAL VESSELS
}

BY

\author{
Ida Mann and James A. Ross
}

LONDON

CARIISLE

IN a paper on " Certain abnormal conditions of the macular region usually classed as colobomata," which appeared in this journal in March, 1927, an attempt was made to classify congenital defects in the neighbourhood of the posterior pole and to provide some diagnostic points by means of which a rough idea of the time of origin and the mechanism of production of the abnormality might be gained.

The anomalies were grouped into three classes (intermediate types also being known to occur), namely,

1. Pigmented macular coloboma.

2. Non-pigmented macular coloboma.

3. Macular coloboma associated with abnormality of blood vessels.

The various theories previously advanced to explain these conditions were discussed, and the balance of evidence was thought to be in favour of the orcurrence of an ante-natal choroiditis as the determining factor. The varying appearances of the different groups were to be explained by the period of foetal life at which this occurred and by the superimposed aberrance of subsequent development which might or would ensue. The earlier the pathological process the more profound would be the modification of subsequent growth and the less would the end result resemble that of a post-natal choroiditis. Of the types of case described, the third (namely, that associated with abnormality of blood vessels) is by far the most rare, and it is in the belief that a further variant of this group has been found that the present case is published. In the paper previously referred to, three cases only were described under the third heading, and in two of these the vascular anomaly took the form of an abnormal vitreous vessel attached to the site of choroidal disturbance. In the third case, however, the anomaly was of a different nature and consisted of an anastomosis between retinal and choroidal vessels in the macular region.

Fig. I shows the appearance of the fundus in this case, which was originally described by Beaumont in 1891 . It will be seen that the site of the macula is occupied by a circular white patch, resembling a second disc, in which the upper temporal branch of 


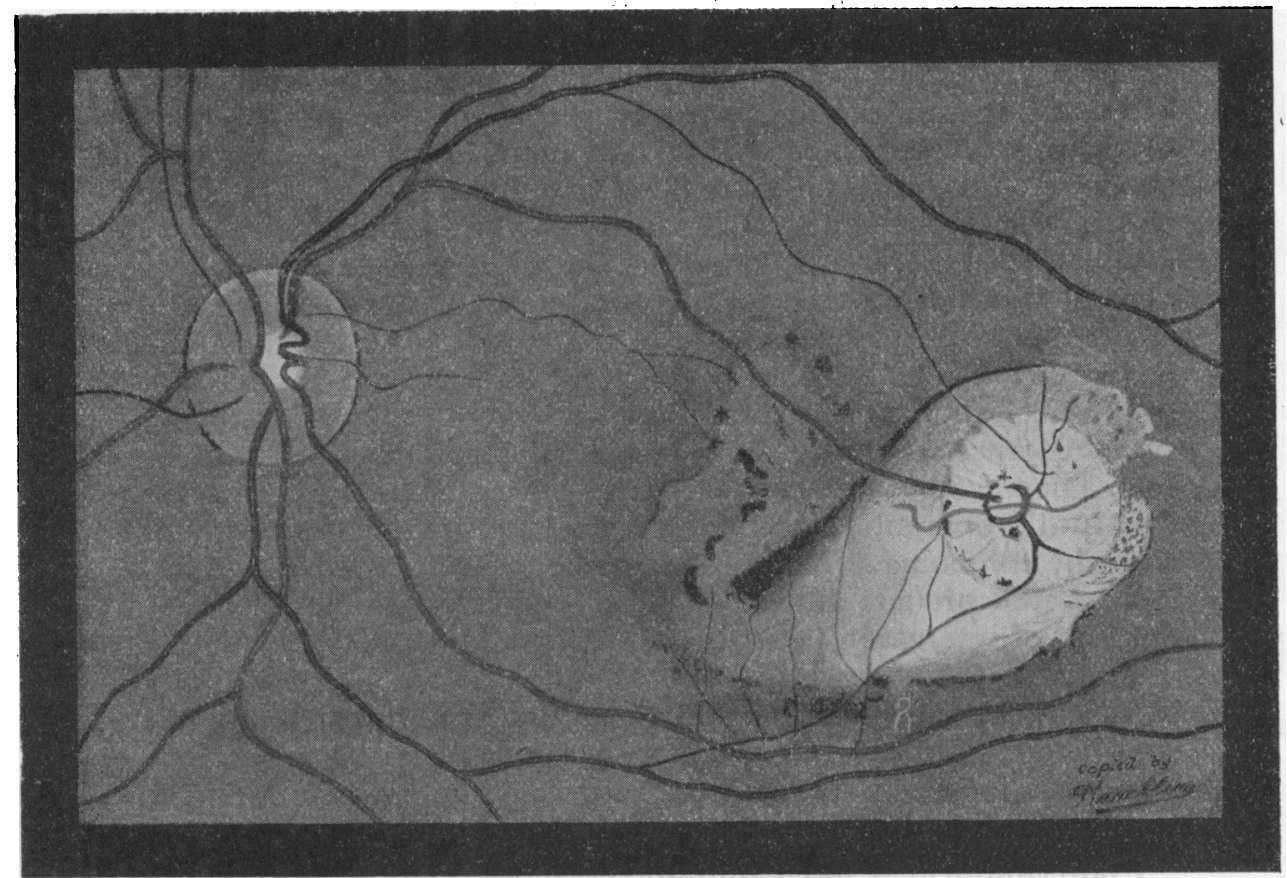

FIG. 1.

Drawing, after Beaumont, of a macular coloboma showing abnormal anastomosis of vessels.

the arteria centralis retinae anastomoses with a choroidal vessel and with a tributary of the lower temporal vein of the retina.

The present case much resembles this, and obviously belongs to the same group. It differs, however, from Beaumont's case in that the coloboma is situated on the temporal side and somewhat above the disc, instead of at the macula, and that the upper temporal quadrant of the retina is supplied almost entirely by vessels from the coloboma and not by the upper temporal branch of the arteria centralis retinae. The case will now be described in detail and the possible embryology of the condition considered.

Clinical history.-The patient, J. R., aged 59 years (labourer), was seen by one of us (J.A.R.) complaining of "watering eyes" -a condition of long duration but worse recently. He is engaged in demolishing old houses and suffers from a catarrhal conjunctivitis of old standing, evidently aggravated by constant working in irritating dust.

R.V. $=6 / 9$, with correction $=6 / 6$.

L.V. $=6 / 12$, with correction $=6 / 9$. 
Routine examination of the fundus revealed the condition shown in Fig. 2. The disc shows an unusual condition of vessels, the superior temporals being represented by a single threadlike vein. $\mathrm{Up}$ and out from the disc a pearly white cup is seen. Its margin overhangs, except to the lower half of its nasal side, where it is shelving. The cup is deep and from it emerge large vessels which supply the upper temporal quadrant of the fundus. Between the disc and the cup (or coloboma) the choroidal vessels are easily seen and the diminutive superior temporal vein anastomoses with them at two points at the nasal edge of the cup. No other anastomosis is found. There is some pigmentary disturbance (consisting of groups of faint greyish dots) here and there around the disc at a short distance from it and not particularly associated with the vessels. The macular area is stippled. Perimetry shows a normal blind spot and (apparently continuous with it) a scotoma corresponding with the coloboma. There is no sector defect and no defect in the area of the arcuate fibres. Colour vision is normal. There is nothing of interest in the patient's personal or family history.

The case, therefore, is one of note and, as far as we know, unique, in that a complete quadrant of the retina (the upper temporal) is supplied by a group of adventitious vessels appearing in the fundus through a localised defect in the retina and chorio-capillaris above and outside the disc.

Embryological Considerations (I.M.).-The question of the possible mechanism whereby such a condition might be produced is an interesting one. There is, we think, no doubt that the anomaly is a congenital one. The clear cut nature of the defect in the retina and the complete absence of any adventitious fibrous tissue, either on the retina or in the vitreous, and of all signs of present or recently past inflammation, preclude the possibility of trauma or post-natal disease, even apart from the vascular abnormality, which latter we feel assured can only be explained on developmental grounds.

The probable sequence of events in the embryonic life of this case appears to be as follows.

A patch of choroiditis (or choroidal disturbance of unknown aetiology) probably occurred, during the fourth month, above and outside the disc. Such a patch would involve the chorio-capillaris layer of the choroid and probably also the external choroidal layer (of tributaries of the venae vorticosae, which become recognisable by the third month) but would not necessarily destroy the intermediate layer (of branches of supply from the short ciliary arteries) which is only just beginning to develop at the posterior pole at four months.

The chorio-capillaris might be completely destroyed and the subjacent scleral condensation (at this period thin, cellular and soft) 
might yield and become slightly ectatic. The overlying retina would also be involved to a certain extent. Its pigment layer and the membrane of Bruch (which appears at the $14 \mathrm{~mm}$. stage) would be destroyed, since these are, from the beginning, closely adherent to the choroid.

The inner layers, however, might escape with much less damage, since they would be separated from the focus of disturbance by the potential cavity of the primary optic vesicle.

The rods and cones would probably suffer, but the layers internal to the external limiting membrane (and more especially the nerve fibre layer, already formed) would not necessarily be completely destroyed. This sparing of the inner layers would explain the absence of any sector-shaped field defect such as one would expect to find if the lesion had involved the whole thickness of the retina after the formation of the nerve fibre layer.

The lesion in the membrane of Bruch has removed the normal barrier which exists here between mesoderm and neural ectoderm and as a result it is quite possible for new arteries, developing from the intermediate layer of the choroid at the margin of the patch, to invade the overlying retina from its deep surface.

Such invading vessels would, at the end of the fourth month, find the retina prepared, if we may use the expression, to receive them, since it is at $100 \mathrm{~mm}$. (16 weeks) that the arteria centralis retinae begins to bud out from the bulb of the hyaloid on the optic disc. The invading arterioles would spread peripherally in the retina and an accompanying system of venules and veins would appear pari passî beside them, just as the tributaries of the vena centralis retinae appear in sit $\hat{u}$ in the normal process of vascularisation.

If this process commenced at the temporal margin of the coloboma immediately before the appearance of vessels at the edge of the optic disc, then it seems reasonable to suppose that the normal development of an upper temporal branch of the arteria centralis retinae would be inhibited, since its area of supply would be already vascularised by the abnormal vessels. That this has occurred seems extremely likely from the appearance of the fundus (in Fig. 2) where one can recognise an abortive upper temporal vein which actually reaches the coloboma and anastomoses with a choroidal vessel in its floor.

The abnormal artery of supply is presumably one of the short posterior ciliaries and might be named a cilio-retinal artery, so long as it be understood that its development is not comparable with that of the usual cilio-retinal vessel seen at the temporal edge of the disc. In the case of this latter all that is required to produce it is the persistence of one of the anastomoses, so common in foetal 
life and in the adult condition in many mammals, between the vessels of the choroid and of the optic nerve round the edge of the disc.

In the present case, however, there is a definite aberrance of growth, in that some abnormal process has destroyed the membrane of Bruch and allowed of an anastomosis between retinal and choroidal vessels in a situation where this never occurs normally in mammalian ontogeny or phylogeny.

The freedom of the rest of the eye from any abnormality and the clear cut nature of the defect, seem to us to point to a single localised lesion at a definite time rather than to any inherent failure in organogeny.

It is in the belief that the explanation can be found in a localised patch of choroidal disturbance occurring during the fourth month and that the case falls into the class of atypical colobomata associated with abnormal vessels that we venture to publish it.

\section{REFERENCES.}

Beaumont, W. M.-Trans. Ophthal. Soc. U.K., Vol. XI, 1891.

Mann, Ida C. -Brit. Jl. of Ophthal., March, 1927.

\section{BUPHTHALMOS AND NAEVUS}

BY

\section{T. R. AynSLEY}

BOURNEMOUTH

I WISH to record four cases of buphthalmos presenting certain features in common. Two of the cases occurred in the course of my own work (E.R. and M.C.) and the other two I was able to examine at the Fountains Hospital.

Case 1. E.R., female, aged 9 years, imbecile. Extensive naevus on the face, scalp, left arm and left side of chest, also some patches on left buttock and thigh. The facial naevus has spread on to the right side of the face but at birth it was said to be confined to the left side. It extends on to the eyelids, but the conjunctiva is not affected. There is a right congenital hemiplegia and an X-ray plate of the skull shows a large calcified meningeal naevus (Fig. 1).

Left eye-cornea enlarged to about $13 \mathrm{~mm}$. Anterior chamber deep. No thinning of the sclera. Some dilated episcleral veins. Pupil reacted sluggishly to light. The iris stroma appeared much denser than in the right eye and the colour was grey green, the other being light blue (Fig. 2). Optic disc large and deeply 